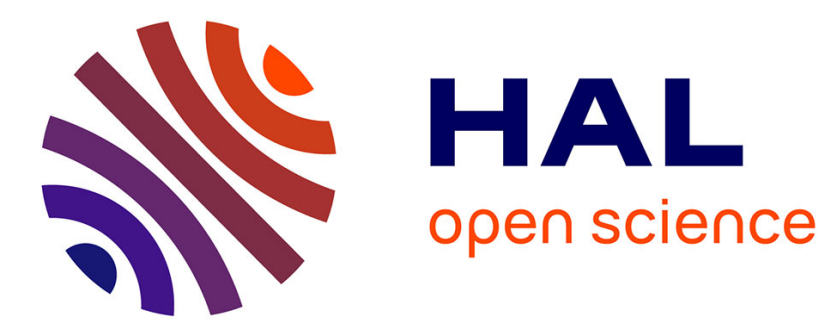

\title{
Les Objectifs du Millénaire pour le développement, l'accès à l'eau et les rapports de genre
}

\author{
Stéphanie dos Santos, Madeleine Wayack Pambè
}

\section{To cite this version:}

Stéphanie dos Santos, Madeleine Wayack Pambè. Les Objectifs du Millénaire pour le développement, l'accès à l'eau et les rapports de genre. Mondes en Développement, 2016, 174 (2), pp.63-78. 10.3917/med.174.0063 . hal-01793945

\section{HAL Id: hal-01793945 \\ https://hal.science/hal-01793945}

Submitted on 17 May 2018

HAL is a multi-disciplinary open access archive for the deposit and dissemination of scientific research documents, whether they are published or not. The documents may come from teaching and research institutions in France or abroad, or from public or private research centers.
L'archive ouverte pluridisciplinaire HAL, est destinée au dépôt et à la diffusion de documents scientifiques de niveau recherche, publiés ou non, émanant des établissements d'enseignement et de recherche français ou étrangers, des laboratoires publics ou privés. 


\title{
Les Objectifs du Millénaire pour le développement, l'accès à l'eau et les rapports de genre
}

\author{
Stéphanie DOS SANTOS ${ }^{1}$ et Madeleine WAYACK PAMBE ${ }^{2}$
}

L'élimination des inégalités sociales de sexe qui découlent de l'accès à l'eau potable est affichée comme une cible prioritaire dans les déclarations internationales des politiques de développement. L'article démontre que, si l'OMD 7C qui visait à "réduire de moitié, d'ici à 2015, le pourcentage de la population n'ayant pas accès de façon durable à un approvisionnement en eau potable" a pu être atteint sur le plan purement statistique, il a eu peu d'effets sur les inégalités de genre dues à l'accès à l'eau.

Mots-clés: Objectifs du Millénaire pour le Développement, accès à l'eau, inégalités, genre

Classification JEL : I31, J16, Q25, Q56

\section{Millennium Development Goals, Access to Water and Gender}

The eradication of gender inequalities arising from access to drinking water is exhibited as a target in many statements of international policy development. The paper demonstrates that even though Millennium Development Goal 7C which aimed to "halve, by 2015, the percentage of the population without sustainable access to safe drinking water" was achieved in terms of quantitative indicators, it has little effect on gender inequalities resulting from access to drinking water

Keywords: Millennium Development Goals, access to water, inequalities, gender

\footnotetext{
${ }^{1}$ Laboratoire Population Environnement Développement (LPED), Institut de recherche pour le développement (IRD) - Aix Marseille Université, en poste à l'Institut Supérieur des Sciences de la Population, Université de Ouagadougou. stephanie.dossantos@ird.fr

${ }^{2}$ Institut Institut Supérieur des Sciences de la Population, Université Ouaga I Professeur Joseph Ki-Zerbo.mwayack@issp.bf
} 
$\mathrm{E}$ n 1990, année qui marque le début de l'observation des Objectifs du Millénaire pour le développement (OMD), 24\% de la population mondiale n'avait pas accès à une source améliorée en eau potable. En 2015, cette proportion était de 9\%. Dans un rapport datant de 2015 qui vise l'évaluation des progrès réalisés dans le cadre des $\mathrm{OMD}$ en matière d'accès à l'eau et d'assainissement ${ }^{3}$, les Nations unies annonçaient que l'OMD 7C qui visait à "réduire de moitié, d'ici à 2015, le pourcentage de la population n'ayant pas accès de façon durable à un approvisionnement en eau potable" était globalement atteint dans le monde. Cet objectif aurait même été atteint depuis 2010, soit cinq ans avant la date butoir fixée, la fin de l'année 2015 (WHO/UNICEF, 2015).

Les questions de gestion et d'accès à l'eau potable sont un thème récurrent dans la sphère du monde du développement depuis au moins un demi-siècle (Langford et Winkler, 2014). Au fil des grandes conférences internationales, les définitions et les indicateurs ont façonné des objectifs différents, révélateurs, à chaque époque, d'une certaine conception du paradigme du développement et des moyens d'y parvenir. Il en est de même de la prise en compte des rapports sociaux de sexe comme une dimension de la problématique de l'eau (Dos Santos, 2012a). À ce titre, la littérature étudiant les conséquences des difficultés d'accès à l'eau sur les femmes est relativement fournie. Notamment, dans une perspective économique, le temps occupé dans la collecte de l'eau est analysé comme une perte de temps ayant un coût économique. Par conséquent, libérer ce temps permettrait-il aux femmes de se consacrer davantage à des activités génératrices de revenus, augmentant les revenus du ménage, ou encore aux soins des enfants et à l'éducation tout particulièrement (Sorenson et al., 2011). Il est toutefois saisissant de constater le décalage notable entre la déclaration de grands principes et leurs mises en action, comme on peut le voir à travers la simple mesure de l'accès à l'eau retenue dans le cadre des OMD. Cet exemple, au cœur de la problématique de cet article, est révélateur de la nécessité d'adopter une vision intégrée, holistique, du développement. D'ailleurs, la page de couverture du dernier rapport de suivi de l'indicateur de l'accès à l'eau et à l'assainissement des OMD paraît pour le moins antinomique : sont représentées une petite fille et une vieille dame, tout sourire, la première portant un seau dont on imagine qu'il contient de l'eau.

Malgré la reconnaissance du rôle de l'accès à l'eau potable dans le développement, près de 700 millions de personnes en demeurent toujours privées en 2015. L'atteinte de cette cible, telle que définie dans ce cadre, est d'ailleurs largement insuffisante pour répondre aux enjeux du développement visés par les différents OMD, surtout en termes de réduction des inégalités sociales. L'objectif de l'article est donc de démontrer que l'accès à l'eau est l'une des clés de voûte de la réduction des rapports sociaux inégaux. Dans une première partie, nous exposerons les limites de l'OMD 7C, tant dans sa

\footnotetext{
${ }^{3}$ Dans cet article, il ne sera question que de l'accès à l'eau.
} 
conception (définition), que dans sa construction (sources de données utilisées), et dans les enjeux auxquels il souhaite répondre. En portant la focale sur les diverses modalités de l'accès à l'eau, par la désagrégation de l'indicateur, cette partie permet de rendre compte des difficultés d'accès d'une part non négligeable de la population mondiale considérée comme ayant accès à cette ressource vitale. La seconde partie aura pour objectif d'apporter un éclairage contextualisé, celui du fardeau de la corvée de l'eau dans les zones informelles de la ville de Ouagadougou au Burkina Faso. L'analyse de données quantitatives spécifiques à ces questions, collectées auprès de 1200 ménages, permettra d'illustrer le décalage entre la mesure d'une statistique et la réalité vécue, particulièrement par les femmes et les enfants, qui sont les principaux gestionnaires de la ressource en eau dans les ménages. Cette étude de cas est centrée sur cette ville où $99 \%$ de la population est considérée comme ayant un accès amélioré à l'eau (INSD et ICF International, 2012). Enfin, une dernière partie sera consacrée à la mise en évidence du rôle central de l'OMD sur l'accès à l'eau dans la réalisation des autres objectifs, montrant l'interdépendance des différents OMD, et soulignant à nouveau son rôle crucial dans le développement des pays.

\section{L'ACCÈS À L'EAU : QUEL OBJECTIF ATTEINT ?}

L'indicateur qui sert de référentiel pour mesurer les progrès réalisés dans le cadre de l'OMD 7C repose sur le taux d'accès de la population à une source d'eau dite "améliorée". Les techniques d'accès à cette source d'eau sont le raccordement de la résidence au réseau d'adduction d'eau par le biais d'au moins un robinet placé dans la cour ou le logement, l'accès à une bornefontaine collective, à une pompe à motricité humaine ou mécanique, à un puits protégé, à une eau de surface protégée et traitée, ou encore la collecte de l'eau de pluie. À l'inverse, les techniques d'accès à l'eau dites non améliorées sont essentiellement les puits non protégés, les eaux de surface non protégées, les vendeurs d'eau ambulants et les citernes d'eau, quelle que soit la provenance de l'eau distribuée par ces deux derniers. Un programme conjoint de l'Organisation mondiale de la santé et de l'UNICEF suivait les progrès de cet indicateur. À partir de sources de données diverses, non spécifiques à la problématique de l'accès à l'eau, ce programme estimait chaque année les progrès réalisés et ceux à réaliser en vue d'atteindre la cible fixée à la fin de l'année 2015. Ces données provenaient principalement des recensements nationaux de la population et de l'habitat, des grandes enquêtes nationales de type enquêtes démographiques et de santé (EDS) ou des enquêtes sur la pauvreté de la Banque mondiale et, récemment, des enquêtes à indicateurs multiples (MICS) que l'UNICEF a mises en place afin de pallier le manque de données dans certains pays, voire de disposer de ses propres bases de données pour le suivi de certains OMD. 
Au terme de la période de suivi des OMD, les Nations unies estiment donc que $91 \%$ de la population mondiale a accès à une source améliorée en eau. Cinq régions en développement ont atteint l'OMD 7C. Les progrès ont surtout été observés en milieu urbain. En 2015, la part de la population urbaine ayant accès à l'eau était de $96 \%$, alors que cette proportion n'était que de $84 \%$ en milieu rural. Cependant, les régions du Caucase, de l'Asie, de l'Afrique du Nord, de l'Océanie et de l'Afrique au sud du Sahara n'ont pas atteint la cible. Au total, en chiffres absolus, 2,6 milliards de personnes qui n'avaient pas accès à une source améliorée en 1990 ont désormais accès à ce type de source en 2015. Les pays ayant connu des progrès remarquables sont, notamment, le Burkina Faso, le Cambodge, le Ghana, la Guinée-Bissau, le Malawi, le Mali, le Paraguay, Vanuatu et l'Ouganda. Dans ces pays, au moins trois personnes sur cinq en 2015 ont pu accéder à une source améliorée depuis 1990, soit, selon le pays, entre 59 et $67 \%$ de la population de 2015. De manière générale toutefois, la catégorie des pays les moins avancés ${ }^{4}$ n'a pas atteint l'OMD $7 \mathrm{C}$, quand bien même $42 \%$ de leurs résidents ont eu accès à l'eau depuis 1990. Au final, dans le monde, une personne sur dix (663 millions d'habitants) est toujours sans accès à une source d'eau améliorée.

Au-delà de cette comptabilité, documentant les progrès d'un côté et les insuffisances de l'autre, se cachent des réalités complexes. En effet, même dans le cas d'améliorations quantitativement mesurées, les populations vulnérables peuvent toujours faire face à des difficultés d'accès. La manière dont l'OMD a été défini et comptabilisé ne prend pas en compte un certain nombre de dimensions de l'accessibilité à l'eau. Il en est ainsi de la qualité du service proposé comme relevant d'un accès amélioré à l'eau qui n'inclut pas la distance, la pénibilité, la continuité du service, la qualité de l'eau délivrée, ou encore le coût. Mais, surtout, sont ignorées les conséquences de ces difficultés d'accès à une source dite améliorée sur le maintien de rapports sociaux inégaux.

Prenons l'exemple de l'accès à l'eau courante, soit un robinet (au minimum) placé dans la cour ou le logement. Ce type d'équipement peut être considéré comme étant celui qui apporte la meilleure garantie de répondre aux différents enjeux sanitaires et sociaux de l'accès à l'eau (Dos Santos, 2012b). C'est l'accès optimal selon certains auteurs : les quantités d'eau par personne sont plus importantes, la qualité est meilleure, il évite la corvée d'aller chercher l'eau à l'extérieur de l'habitation, pour un rapport qualité du service/prix globalement parmi les meilleurs (Howard et Bartram, 2003). D'ailleurs, c'est l'accès à "l'eau à tous les étages" qui est une des explications fortes de la baisse de la mortalité observée dans les pays occidentaux des XIXème et XXème siècles, notamment en France (Preston et Van de Walle, 1978) ou aux États-Unis (Cutler et Miller, 2005). À bien des égards, ce type d'accès à l'eau devrait-il être considéré comme une cible per se à atteindre. Or, dans le monde, deux personnes sur cinq n'ont

${ }^{4}$ Il s'agit d'une liste de 48 pays, dont une majorité est située en Afrique, les autres en Asie, dans les Caraïbes et en Océanie (cf. http://donnees.banquemondiale.org/region/LDC). 
toujours pas accès à un robinet d'eau dans leur résidence (tableau 1). Cette proportion de non-accès est plus importante en milieu rural qu'en milieu urbain, avec respectivement 7 ruraux sur 10 et 1 citadin sur 5 qui n'ont pas accès à l'eau courante à domicile. Dans les pays les moins avancés, avoir accès à un robinet dans la résidence est quasiment un luxe puisque $88 \%$ de la population n'y pas accès, dont $97 \%$ des résidents en milieu rural dans ces pays.

Tableau 1 : Évolution de la part de la population ayant accès à un robinet dans la résidence, selon le milieu de résidence, pour quelques régions du monde ou pays $(1990-2015$, en $\%)$

\begin{tabular}{|l|c|c|c|c|}
\hline & Année & Milieu urbain & Milieu rural & Ensemble \\
\hline Ensemble du monde & 1990 & 79 & 18 & 44 \\
& 2015 & 79 & 33 & 58 \\
\hline Pays développés & 1990 & 97 & 79 & 92 \\
& 2015 & 98 & 89 & 96 \\
\hline Pays en développement & 1990 & 68 & 11 & 31 \\
& 2015 & 72 & 28 & 49 \\
\hline Pays les moins avancés & 1990 & 29 & 2 & 7 \\
& 2015 & 32 & 3 & 12 \\
\hline Afrique subsaharienne & 1990 & 43 & 4 & 15 \\
& 2015 & 33 & 5 & 16 \\
\hline Afrique du Nord & 1990 & 86 & 33 & 59 \\
& 2015 & 92 & 78 & 86 \\
\hline Asie du Sud-Est & 1990 & 42 & 5 & 17 \\
& 2015 & 51 & 17 & 33 \\
\hline Océanie & 1990 & 74 & 11 & 27 \\
& 2015 & 74 & 11 & 25 \\
\hline Haïti & 1990 & 24 & 1 & 8 \\
& 2015 & 13 & 5 & 10 \\
\hline Kenya & 1990 & 55 & 10 & 17 \\
& 2015 & 45 & 14 & 22 \\
\hline République Démocratique du Congo & 1990 & 48 & 1 & 15 \\
& 2015 & 17 & 1 & 8 \\
\hline Zimbabwe & 1990 & 98 & 7 & 33 \\
& 2015 & 74 & 5 & 28 \\
\hline
\end{tabular}

Source : WHO/UNICEF, 2015.

Le développement économique des nations étant supposé répondre aux attentes d'amélioration des conditions de vie des populations, on devrait observer que la part de la population ayant accès à l'eau courante à domicile a augmenté avec le temps. Cependant, cette part est restée stable pour l'ensemble du monde urbain avec 79\% de la population en 1990 et en 2015. Dans certaines zones urbaines du monde, la part de la population ayant accès à l'eau courante a baissé, comme dans les villes d'Afrique subsaharienne où l'accès est passé de 43\% en 1990 à 33\% en 2015. Des pays ont vu l'accès à l'eau courante en milieu urbain chuter brutalement entre ces deux dates, comme en Haïti ou en 
République Démocratique du Congo (RDC) dont le taux d'accès à l'eau courante a été divisé par plus de deux.

L'instabilité politique, les catastrophes climatiques, voire les deux combinées, peuvent expliquer la baisse de l'accès à l'eau dans certains pays, et à l'eau courante à domicile en particulier, comme c'est le cas pour Haïti ou la RDC. Une autre explication vient par ailleurs de la croissance urbaine mal maitrisée, la croissance démographique dépassant les capacités de la planification urbaine. C'est notamment le cas du Kenya. Pour ce dernier, entre 1990 et 2015, la part de la population urbaine n'ayant pas accès à une source d'eau améliorée est passée de $92 \%$ à $82 \%$, celle de l'accès à un robinet au sein de la résidence de $55 \%$ à $45 \%$. Tout particulièrement, Nairobi, la capitale, a connu un taux de croissance de sa population supérieur à $4 \%$ par an ces dernières décennies, croissance qui s'est accompagnée de l'établissement d'une part croissante de la population dans les bidonvilles. Aujourd'hui, on estime que $60 \%$ de la population de cette capitale d'Afrique de l'Est vit dans les quartiers informels, sur $5 \%$ de la superficie de l'agglomération. Ces quartiers absorberaient, en outre, $75 \%$ de la croissance urbaine de la ville (UN-HABITAT, 2005).

Cependant, les chiffres d'accès à l'adduction d'eau à domicile ne font état que de la présence d'un équipement dans la résidence. Ils ne documentent en rien la qualité du service, par exemple en termes de débit et de continuité. En effet, dans de nombreuses villes des pays en développement, les coupures d'eau sont de véritables stratégies de gestion de la pénurie de l'eau. Par exemple, dans une ville comme Léon, au Nicaragua, alors que l'ensemble des ménages est directement connecté au système d'adduction d'eau, il a été montré que $80 \%$ des ménages disposent d'un système de stockage de l'eau, illustrant de manière latente un problème de continuité quotidienne du service d'eau (Vasquez, 2012). Néanmoins, le stockage de l'eau est l'une des principales sources de contamination de l'eau de boisson dans les ménages, quand bien même l'eau délivrée avant le stockage est potable (Wright et al., 2004). La discontinuité du service peut également avoir pour conséquence d'aller chercher l'eau à l'extérieur de l'habitation, corvée habituellement dévolue aux femmes et aux enfants. Aussi, dans bien des contextes, le taux de couverture de l'accès à l'eau n'est pas suffisant pour illustrer les progrès dans ce domaine. Il doit être couplé à des critères de qualité du service, sans quoi les impacts ciblés seront manqués. Pour pallier le manque d'accès à un robinet dans la cour ou le logement, ou une coupure dans le service d'adduction d'eau, une des alternatives relativement fréquente dans les pays en développement repose sur le recours à un revendeur livrant l'eau à domicile. Lorsqu'un ménage peut financièrement recourir à ce service, la corvée d'eau est alors réduite pour les membres du ménage. Bien souvent, les vendeurs s'approvisionnent aux bornes-fontaines collectives, plutôt qu'aux pompes à motricité humaine ou aux puits. Ce mode d'accès à l'eau est toutefois classé comme un accès non amélioré à l'eau, en raison principalement du surcoût payé par les ménages qui font appel à leur service. La définition de l'OMD 7C inclut, en effet, un certain nombre de critères comme le coût économique de l'eau, qui ne doit pas constituer une part trop importante des 
revenus du ménage, et la durabilité du service, critères fondés sur des postulats et qui ne sont en rien mesurés dans le cadre du suivi de l'OMD (pour des détails, cf. Dos Santos, 2012b). La qualité microbiologique de l'eau fournie par les revendeurs à domicile est aussi avancée comme un élément contribuant à catégoriser ce type d'accès comme non amélioré. Pourtant, une revue de littérature a montré qu'il n'existe pas de preuves significatives que la qualité de l'eau fournie pas ces revendeurs est de mauvaise qualité (Opryszko et al., 2009). La littérature a même montré que l'eau provenant des revendeurs peut être de meilleure qualité par rapport à celle provenant de certaines sources dites améliorées. En effet, l'eau revendue par les marchands à domicile provient à l'origine d'une source d'eau traitée chimiquement, souvent des bornes-fontaines, ce qui lui assure le maintien d'une certaine qualité durant deux à trois jours grâce au chlore résiduel et ce, contrairement aux eaux provenant de forages, de pompes ou de puits protégés qui ne sont généralement pas traitées à la source (Ferguson et al., 2011). Si l'on peut admettre que l'eau délivrée à domicile par les revendeurs n'est pas un mode d'accès à privilégier, et donc à classer comme amélioré, du seul fait du coût de ce service privé, on peut toutefois s'étonner de sa quasi-absence des statistiques servant de base au suivi de l'indicateur. Dans certaines villes sahéliennes, ce mode d'accès peut être très répandu, comme à Niamey, la capitale du Niger. Ce pays, classé dernier sur 188 pays au regard de l'indicateur de développement humain de 2015 (UNDP, 2015), affiche un taux d'accès à une source d'eau améliorée de 100\% en milieu urbain (WHO/UNICEF, 2015). Ce constat est pour le moins surprenant si on le compare à l'ensemble des pays les moins avancés où ce même taux est de $86 \%$. Cependant, il semble qu'il y ait un acteur essentiel des services d'eau qui a disparu du comptage des OMD : les Garoua ("vendeurs d'eau» en langue haoussa) qui délivrent l'eau à domicile, notamment dans les quartiers informels où ils approvisionneraient une grande majorité de la population (Nassartenaye, 2011). Cet exemple illustre la manière dont cet indicateur, et les sources de données utilisées, sont pour le moins sujets à caution, car ils surestiment les taux d'accès, et négligent les coûts sociaux et économiques induits par la corvée d'eau.

Ceci est vérifié lorsque l'on s'intéresse à une modalité particulière de l'accès à l'eau, l'accès à l'extérieur de la résidence. Lorsqu'un ménage ne dispose pas d'au moins un robinet dans sa cour ou son logement, et qu'il ne fait pas appel à un revendeur livrant l'eau à domicile, l'eau nécessaire aux besoins domestiques de base doit être collectée à une source distante du domicile. Sur ce point, la définition elle-même est opaque, puisque l'accès à une source d'eau améliorée doit être à une distance "convenable», sans précision sur ce que cet adjectif sousentend réellement. Or, au Cambodge, alors que 100\% de la population urbaine a accès à une source d'eau améliorée selon l'OMD, pour $28 \%$ de celle-ci, le point d'approvisionnement est situé à une distance qui nécessite 30 minutes de temps de trajet au maximum (et 1,3\% à plus de 30 minutes) selon l'EDS de 2014. De la même manière, à Bamako, 97\% de la population aurait accès à l'eau, alors que $40 \%$ de la population doit consacrer jusqu'à 30 minutes de temps de trajet pour s'approvisionner (6\%, plus de 30 minutes) selon l'EDS de 
2012. Des données fines, permettant d'évaluer précisément les distances pour la ville de Ouagadougou, indiquent qu'entre 35 et $50 \%$ de la population résidant dans les quartiers informels doit parcourir entre 10 et 30 minutes pour accéder au point d'approvisionnement amélioré. Dans ces ménages, l'eau domestique nécessite de ce fait d'être collectée par une ou plusieurs personnes du ménage qui consacrent un certain temps de trajet jusqu'au point d'eau, de même qu'un certain temps d'attente sur place, ce dernier n'étant d'ailleurs pas pris en compte dans la définition de l'OMD. L'eau doit ensuite être transportée puis stockée dans des contenants qui nécessitent eux-mêmes quelques manipulations et mesures d'entretien.

Du fait du rôle social assigné à chaque sexe au sein de l'institution familiale, il existe une inégale répartition des tâches entre les hommes et les femmes. De nombreux travaux ont montré que les activités de reproduction ${ }^{5}$ dans la famille, dont fait partie la corvée d'eau, sont encore largement dévolues aux personnes de sexe féminin). Dans les pays en développement, la collecte des ressources nécessaires aux besoins domestiques de base (l'énergie pour la cuisson des aliments ou le chauffage, les légumes, les fruits, l'eau, etc.) est particulièrement consommatrice en temps et en énergie. Dans certaines régions en Inde, la participation des femmes à ces activités est six fois supérieure à celle des hommes (Hirway et Jose, 2011). Dans les ménages africains qui s'approvisionnent en eau à l'extérieur de leur résidence, la corvée d'eau reflète aussi la division du travail selon le genre au sein des ménages (Dos Santos, 2012a). Par conséquent, c'est habituellement aux femmes et aux filles qu'elle revient. En Afrique subsaharienne, on estime que les femmes et les filles passeraient quelques 40 milliards d'heures par an à transporter de l'eau (PNUD, 2006, 48). Ces activités constituent alors une forte contrainte dans l'organisation de l'emploi du temps des femmes. Cette inégalité sociale peut être analysée à partir du concept de pauvreté en temps (Garhammer, 1998), bien qu'il fut d'abord développé dans les pays du Nord. Ces tâches domestiques rentrent en compétition avec la possibilité de développer des activités économiques ou de formation, limitant la capacité à profiter des opportunités économiques (Blackden et Wodon, 2006).

Toutefois, que l'eau collectée à l'extérieur de l'habitation provienne d'une bornefontaine, d'une pompe à motricité humaine ou d'un puits protégé par une margelle, le ménage est considéré par l'indicateur des OMD comme bénéficiant d'un accès amélioré à l'eau, à l'instar de la situation confortable où l'eau domestique provient directement, et sans effort, d'un ou de plusieurs robinets situés dans les pièces où ils sont nécessaires. On s'interroge ici sur l'équité sociale de l'indicateur, qui considère comme équivalents différents types

\footnotetext{
${ }^{5}$ Ce concept renvoie à l'ensemble des activités domestiques, réalisées au sein de la famille, non rémunérées, et qui ont pour rôle la reproduction sociale de la cellule familiale. D'un autre côté, les activités productives sont souvent associées au travail rémunéré qui permet à la famille de subvenir à ses besoins matériels.
} 
d'approvisionnement n'impliquant pas les mêmes contraintes. Et ce, d'autant plus que cette iniquité induit une persistance des configurations des pratiques de genre, comme le montre le cas de la corvée d'eau dans les quartiers informels de la ville de Ouagadougou.

\section{LE FARDEAU DE LA CORVÉE D'EAU DANS LES QUARTIERS INFORMELS DE OUAGADOUGOU}

En 2010, 99\% de la population de Ouagadougou aurait eu accès à une source d'eau améliorée (INSD et ICF International, 2012). Une analyse récente de la corvée de l'eau dans les zones informelles de la ville a toutefois montré comment, en ne s'en tenant qu'à cette seule mesure statistique, les inégalités sociales étaient profondément occultées, et notamment celles relevant des rapports de genre, (Dos Santos et Wayack-Pambe, 2015). Les données mises en valeur dans cette analyse ont été recueillies lors de deux enquêtes quantitatives. La première date de février 2013. Elle a été réalisée auprès de 1200 ménages représentatifs des trois quartiers informels de l'Observatoire de population de Ouagadougou $(\mathrm{OPO})^{7}$ que sont Nioko 2, Nonghin et Polesgo. Le questionnaire utilisé dans cette enquête comportait plusieurs modules renseignant, entre autres, les modalités de l'accessibilité à l'eau, intégrant des précisions sur la collecte de l'eau (qui en a la charge ?, avec quels récipients ?, quel moyen de locomotion ?, etc.). Les caractéristiques des personnes en charge de la collecte de l'eau au sein du ménage ont ensuite été retrouvées à partir de la base de données du système de suivi de l'OPO.

La deuxième enquête a eu lieu en décembre 2013 auprès d'une population de 211 femmes âgées de 15 à 66 ans, issues des ménages concernés par la première enquête. Ces femmes avaient été précédemment repérées comme étant les premières responsables de la collecte de l'eau dans leur ménage. Cette deuxième phase d'enquête visait à mesurer l'emploi du temps de ces femmes en charge de la collecte de l'eau, de même que les opportunités potentielles si elles n'avaient pas à collecter l'eau à l'extérieur de l'habitation. Les modules servant à recueillir ces données ont été développés en référence au guide de production de mesures d'emploi du temps, réalisé par le département des affaires économiques et sociales des Nations unies (United Nations, 2005).

6 De manière générale, la notion de quartiers informels fait référence à un type d'établissement humain spontané, les populations installant leurs habitations sans autorisation officielle de la part des autorités municipales. À Ouagadougou, cette occupation des sols se fait toutefois avec l'accord du chef coutumier, moyennant une somme variable selon le quartier et l'emplacement de la parcelle, notamment par rapport à la voie bitumée. Ces quartiers, appelés aussi non lotis dans cette ville, se caractérisent par un manque d'accès aux services urbains de base comme l'eau, la voirie, l'électricité. Un tiers des ménages de la ville résideraient dans les quartiers non lotis à Ouagadougou (Boyer et Delaunay, 2009).

${ }^{7}$ Pour des détails sur l'Observatoire de population de Ouagadougou, on pourra se reporter à Rossier et al. (2012) 
Tout d'abord, les résultats ont confirmé le fait que l'absence de l'eau courante dans un ménage implique une très forte mobilisation des personnes de sexe féminin pour la collecte de l'eau à l'extérieur de la résidence. Dans les ménages enquêtés qui ne disposent pas d'un raccordement au réseau d'adduction d'eau, la personne principalement en charge de la collecte de l'eau est de sexe féminin dans $83,6 \%$ des cas. La réalisation de cette tâche mobilise davantage les petites filles que les petits garçons, puisque près d'une personne sur dix principalement chargée de la collecte d'eau est une fille âgée de moins de 16 ans, contre 3\% pour un garçon du même âge. De même, les personnes de sexe féminin sont surreprésentées parmi les membres du ménage qui secondent dans cette tâche la personne principalement chargée de la collecte d'eau, avec $65 \%$ des aides à la corvée d'eau apportées par des personnes de sexe féminin, contre $35 \%$ de sexe masculin.

La pénibilité des tâches dédiées à la collecte de l'eau au quotidien se différencie également selon le genre. Concernant les types d'approvisionnement en eau, il ressort dans les quartiers informels enquêtés que les personnes de sexe masculin chargées de la collecte de l'eau domestique s'approvisionnent souvent aux bornes-fontaines et les personnes de sexe féminin aux forages, alors que ces derniers nécessitent un effort physique important du fait d'une extraction de l'eau par pompage manuelle ou à pied. Parmi les individus de plus de 26 ans révolus en charge de la collecte de l'eau, le forage constitue le mode principal d'approvisionnement chez les femmes dans 35\% des cas, contre seulement 14\% chez les hommes. Cette préférence des femmes pour ce type d'approvisionnement s'explique à la fois par des facteurs économiques (l'eau aux forages est moins chère que l'eau aux bornes-fontaines) et par la distance. En effet, les femmes disposant moins souvent de moyens de transports motorisés que les hommes, elles privilégient davantage les forages qui sont souvent moins éloignés des habitations des zones informelles que les bornesfontaines situées dans les zones formelles). Le temps d'attente aux points d'eau est un autre élément important révélateur de la pénibilité de la collecte de l'eau. Il est dû aux longues files d'attente pour collecter l'eau, celles-ci étant la résultante, soit des coupures d'eau ou de la faible pression de l'écoulement aux bornes-fontaines, soit de la densité de population desservie par le point d'eau. Les temps d'attente aux bornes-fontaines sont, en moyenne, moins longs qu'aux forages, la collecte de l'eau étant facilitée par l'adduction d'eau, même si parfois la pression au robinet peut être faible. Les résultats montrent un temps d'attente supérieur pour les personnes de sexe féminin que le temps d'attente pour les personnes de sexe masculin, avec $14 \%$ des femmes qui attendent moins d'une heure au point d'eau et $39,2 \%$ plus de trois heures, contre, respectivement pour les hommes, $36,4 \%$ et $21,4 \%$.

Une des conséquences importantes sur les inégalités sociales de genre de l'absence de l'adduction d'eau dans le ménage est le coût d'opportunité en temps que cela représente pour les personnes de sexe féminin en charge de l'approvisionnement en eau. L'analyse du poids des activités domestiques relatives à la corvée d'eau sur l'emploi du temps quotidien des femmes révèle 
que le morcellement des activités est consommateur en temps, outre le fait d'accroître la pénibilité de la tâche. L'étude d'une journée type montre que les femmes ne sont pas accaparées par cette activité de façon continue, ce qui résulte en une dilution de leur temps, qui les empêche de se consacrer notamment à des activités génératrices de revenus. Par exemple, du fait des longues files d'attente, et parce qu'elles transportent généralement l'eau dans des récipients de faible capacité, les femmes peuvent avoir à effectuer de nombreux aller-retour entre le point d'approvisionnement en eau et leur résidence.

Par ailleurs, ces analyses ont montré que le temps consacré par les femmes à la corvée de l'eau occupe au minimum une heure de leur emploi du temps quotidien, pour près des deux tiers des femmes enquêtées, et entre deux à trois heures pour un quart des femmes. Il apparait donc qu'une femme sur dix utilise plus de $20 \%$ de son temps d'activité à la collecte et à la gestion de l'eau. Aussi, lorsqu'elles sont interrogées sur ce qu'elles feraient, éventuellement, du gain de temps provenant d'une suppression de la corvée de la collecte d'eau, les femmes répondent majoritairement qu'elles consacreraient ce temps à des activités génératrices de revenus ou à d'autres activités domestiques.

Ces quelques résultats empiriques, issus d'enquêtes spécifiques sur la question de l'accès à l'eau, démontrent la nécessité de mesurer finement les conditions réelles d'accessibilité à l'eau, sous peine d'occulter les inégalités dans les rapports sociaux de genre dans l'accès à cette ressource essentielle, mais également de miner les efforts à réaliser dans les autres domaines, le développement étant le résultat de l'interdépendance de différents domaines et acteurs.

\section{L'ACCÈS À L'EAU AU CEEUR DU DÉVELOPPEMENT HUMAIN}

La littérature à la fois théorique et empirique ne manque pas pour démontrer qu'un meilleur accès à l'eau est tout à la fois un moteur de développement et un résultat de celui-ci. Il existe ainsi une corrélation positive entre le niveau de développement d'un pays et le taux d'accès à l'eau. Dans une analyse portant sur 18 pays, Hewett et Montgomery (2001) ont mis en évidence le lien entre l'accès à l'eau et le PIB. Le manque d'accès à ce service de base affaiblit les capacités de croissance économique et sociale et entrave les efforts de réduction de pauvreté, pour les femmes en particulier (Seager, 2010). Les difficultés d'accès à l'eau d'un pays freinent son développement au niveau économique, avec comme enjeux une augmentation des coûts de production sur l'agriculture et l'industrie, par exemple (Davis et al., 2001). Aussi, à bien des égards, l'accès à l'eau est au cœur même du développement, de surcroitt imbriqué dans les autres OMD.

En effet, le lien d'interdépendance avec les objectifs de réduction de la pauvreté (OMD 1) et de l'augmentation de la scolarisation des enfants (OMD 2) est en 
premier lieu évident de par son impact sur les opportunités socio-économiques des individus, des femmes et des filles en particulier.

Les preuves empiriques ne manquent pas pour démontrer que ce sont surtout les petites filles qui sont sollicitées pour aller chercher l'eau à l'extérieur de l'habitation (Dos Santos et Wayack-Pambe, 2015 ; Hirway et Jose, 2011 ; Truelove, 2011 ; Boone et al., 2010 ; Crow et McPike, 2009. Il est donc à ce point étonnant que l'OMD sur l'accès à l'eau ne visait que la réduction de moitié du nombre de personnes n'ayant pas accès à l'eau, alors que parallèlement, l'objectif sur l'éducation se rapportait à l'ensemble des enfants en âge d'aller à l'école primaire.

Un autre paradoxe relatif à la logique de définition des objectifs de développement apparait dans l'OMD 3 qui ambitionne de promouvoir l'égalité de genre et l'émancipation des femmes. En effet, si l'OMD sur l'accès à l'eau avait eu pour objectif de favoriser l'égalité de genre et la promotion des femmes, la définition de l'indicateur aurait dû être davantage contraignante en termes de distance à parcourir, de temps d'attente au point d'eau, de pénibilité de la tâche. En ce sens, l'objectif à atteindre aurait dû cibler l'accès à domicile. L'absence de données sexospécifiques sur ces questions est révélatrice du peu de considération concrète pour les questions de genre dans le domaine de l'eau, en dehors d'une rhétorique pourtant très abondamment utilisée (Seager, 2010). Nous ne reviendrons pas sur les liens, abondamment démontrés dans la littérature (Tarrass et Benjelloun, 2012) entre l'accès à l'eau et la santé des enfants, celle des mères et de tous les adultes en général. Cette problématique des maladies liées à l'eau relie ainsi l'OMD sur l'accès à l'eau à trois autres objectifs relatifs à la réduction de la mortalité des enfants (OMD 4), à l'amélioration de la santé maternelle (OMD 5) et à la réduction des grandes endémies (OMD 6).

En revanche, une dernière interrelation nous semble importante à souligner, celle avec l'amélioration des conditions de vie dans les quartiers informels (OMD 7D). En effet, dans le monde, la population urbaine devrait augmenter de plus des deux tiers d'ici à 2050 , et $90 \%$ de cette augmentation sera le fait des villes africaines et asiatiques. En Afrique tout particulièrement, la population urbaine devrait tripler entre 2020 et 2050 (United Nations, 2014). La croissance urbaine de ces villes se fait pour une bonne part dans des quartiers informels, non aménagés, qui ne bénéficient pas de l'accès aux services urbains de base, dont de l'accès à l'eau. À Nairobi, par exemple, la population vivant dans les bidonvilles devrait doubler d'ici 15 ans (UN-HABITAT, 2005). Dans ces conditions de croissance, vouloir réduire la proportion de citadins vivant dans des taudis (dont le manque d'accès à l'eau est un des critères de la définition du taudis), nécessite une mesure fine des conditions d'habitat dans ces contextes précis, mesure qui semble absente si l'on prend les exemples évoqués ci-dessus de la ville de Niamey ou de Ouagadougou. Il s'agirait alors de repenser la dichotomie classique utilisée dans la présentation des grands indicateurs de développement, basée sur la distinction entre le milieu rural d'un côté et le milieu urbain de l'autre. Sans juger qu'elle soit obsolète, cette dichotomie cache 
cependant une myriade de situations et d'inégalités fortes que seule une désagrégation fine peut mettre au jour. Eu égard à la croissance urbaine à venir, il serait opportun de désagréger la catégorie "milieu urbain", au minimum en distinguant les zones formelles des zones informelles, voire en intégrant la distinction entre le centre et la périphérie, qui ne rejoint pas nécessairement la dichotomie formelle/informelle, et selon les configurations spécifiques de croissance urbaine propre à chaque contexte, entre étalement spatial spontané et paupérisation des centres. L'indicateur d'accès à l'eau, comme l'ensemble des mesures du développement, devraient documenter finement les ségrégations socio-spatiales qui se donnent à lire sans équivoque dans certaines villes, comme à Accra (Songsore et McGranahan, 2002) ou à Ouagadougou (Dos Santos, 2015).

L'accès à l'eau est ainsi un sujet multi-facette, à la fois facteur de développement social et sanitaire, bien économique, environnemental et donc politique. Aussi, à l'instar de Langford et Winkler (2013, 4), nous pensons que la perte de la dimension holistique de l'accès à l'eau est le prix à payer d'une cible floue à atteindre, surtout en l'absence de données sociales précises, comme celles de genre.

\section{CONCLUSION}

Au seuil du XXIème siècle, il reste encore dans le monde près d'une personne sur dix qui n'a toujours pas accès à une source d'eau améliorée, surtout parmi les plus pauvres et/ou résidant en milieu rural. Les continents où l'accès à l'eau est le moins bon sont l'Océanie et l'Afrique subsaharienne. Sans toutefois nier les progrès réalisés dans certaines autres régions du monde depuis les années 1990, l'un des objectifs de cet article était de mettre en exergue les contextes où l'accès à l'eau est particulièrement problématique, y compris dans le cas où les populations sont considérées par la communauté internationale comme ayant accès à une source d'eau améliorée.

Nous avons montré en quoi l'Objectif du Millénaire pour le développement dédié à l'accès à l'eau pouvait être, à bien des égards, un leurre, alors qu'il est la base sur laquelle reposent les autres dimensions du développement. Éthiquement, mais également conceptuellement, on s'étonne encore de constater que ce droit fondamental ne soit toujours pas pensé comme un prérequis au développement.

Il faudrait prendre garde à ce que cette arithmétique du développement ne prenne pas l'ascendant sur les politiques de développement, qui doivent davantage recentrer les populations et les modalités qualitatives de ces indicateurs au cœur même des actions à mener. Que cette "gouvernance par les nombres » (Supiot, 2015), cette réduction des êtres humains à un simple chiffre de performances, d'objectifs quantitatifs à atteindre, n'en fasse pas oublier les enjeux premiers des politiques de développement, à savoir la réduction effective des inégalités sociales, dont celles relatives aux rapports de genre. C'est là où les 
sciences de la société, critiques, nourries d'une démarche de déconstruction de l'objet, ici le chiffre, adoptant un point de vue micro basé sur l'observation, et non pas seulement sur la mesure, doivent prendre toute leur place dans le débat, en apportant une contribution éclairée pour une action publique efficace.

\section{BIBLIOGRAPHIE}

BLACKDEN C. M., WODON Q. (2006) Gender, Time Use, and Poverty in Sub-Saharan Africa, Washington, D.C., World Bank, Working Paper $\mathrm{n}^{\circ} 73$.

BOONE C., GLICK P., SAHN D. E. (2010) Household Water Supply Choice and Time Allocated to Water Collection: Evidence from Madagascar, Journal of Development Studies, vol. 47, n 12, 1826-1850.

BOYER F., DELAUNAY D. (2009) Onaga 2009 - Peuplement de Ouagadougon et Développement urbain. Rapport provisoire, Ouagadougou, IRD - Ambassade de France au Burkina Faso, 250 p.

CROW B., MCPIKE J. (2009) How the drudgery of getting water shapes women's lives in low-income urban communities, Gender, Technology and Development, vol. 13, $\mathrm{n}^{\circ} 1$, 43-68.

CUTLER D., MILLER G. (2005) The role of public health improvements in health advances: the twentieth-century United States, Demography, vol. 42, n 1, 1-22.

DAVIS J., KANG A., VINCENT J. (2001) How important is improved water infrastructure to microenterprises? Evidence from Uganda, World Development, vol. $29, \mathrm{n}^{\circ} 10,1753-1767$.

DOS SANTOS S. (2015) L'accès à l'eau des populations dans les quartiers informels de Ouagadougou : un objectif loin d'être atteint, in A. B. Soura, S. Dos Santos, F. C. Ouédraogo (dir.) Climat et accès aux ressources en eau en zones informelles de Ouagadongou, Ouagadougou, Presses universitaires de Ouagadougou, 57-72.

DOS SANTOS S. (2012a) Le rôle des femmes selon la GIRE : regard sur le troisième principe de Dublin en Afrique au sud du Sahara, in F. Julien (dir.) La gestion intégrée des ressources en eau en Afrique subsabarienne. Paradigme occidental, pratiques africaines, Montréal, Presses de l’Université du Québec, 135-164.

DOS SANTOS S. (2012b) L'accès à l'eau en Afrique subsaharienne : la mesure est-elle cohérente avec le risque sanitaire ?, Environnement, Risques et Santé, vol. 11, n 4, 282 286.

DOS SANTOS S., WAYACK-PAMBE M. (2015) Le fardeau de la corvée d'eau : différenciation de genre et potentiel frein à l'émancipation féminine en milieu urbain informel, in A. B. Soura, S. Dos Santos, F. C. Ouédraogo (dir.) Climat et accès aux ressources en eau en zones informelles de Onagadougou, Ouagadougou, Presses universitaires de Ouagadougou, 93-112.

FERGUSON A. S., MAILLOUX B. J., AHMED K. M., VAN GEEN A., MCKAY L. D., CULLIGAN P. J. (2011) Hand-pumps as reservoirs for microbial contamination of well water, Journal of $W$ ater and Health, vol. 9, $\mathrm{n}^{\circ} 4,708-717$.

FLORO M. S., KOMATSU H. (2011) Gender and Work in South Africa: What Can Time-Use Data Reveal?, Feminist Economics, vol. 17, n 4, 33-66. 
GARHAMMER M. (1998) Time Poverty in Modern Germany, Society and Leisure, vol. 21, 327-352.

HEWETT P., MONTGOMERY M. (2001) Poverty and public services in developing-country cities, New York, Population Council, Policy Research Division, 67 p.

HIRWAY I., JOSE S. (2011) Understanding Women's Work Using Time-Use Statistics: The Case of India, Feminist Economics, vol. 17, n 4, 67-92.

HOWARD G., BARTRAM J. (2003) Domestic Water Quantity, Service Level and Health, Geneva, WHO, 33 p.

INSD, ICF INTERNATIONAL (2012) Enquête Démographique et de Santé et à Indicateurs Multiples du Burkina Faso 2010, Calverton, Maryland, USA, Institut National de la Statistique et de la Démographie, ICF International, $325 \mathrm{p}$.

LANGFORD M., WINKLER I. T. (2014) Muddying the Water? Assessing Targetbased Approaches in Development Cooperation for Water and Sanitation, Journal of Human Development and Capabilities, vol. 15, n 2-3, 247-260.

LANGFORD M., WINKLER I. T. (2013) Quantifying Water and Sanitation in Development Cooperation: Power or Perversity?, Boston, Harvard School of Pulbic Health.

MARPHATIA A. A., MOUSSIÉ R. (2013) A question of gender justice: Exploring the linkages between women's unpaid care work, education, and gender equality, International Journal of Educational Development, vol. 33, $\mathrm{n}^{\circ}$ 6, 585-594.

NASSARTENAYE N. (2011) Accès à l'eau potable et à l'assainissement; quels enjeux pour la santé dans les quartiers précaires? Étude appliquée au quartier Gamkallé de la commune IV de Niamey au Niger, Niamey, mémoire de maîtrise de géographie, Université Abdou Moumouni de Niamey.

OPRYSZKO M. C., HUANG H. K. S., SCHWAB K. J. (2009) Data gaps in evidencebased research on small water enterprises in developing countries, Journal of water and bealth, vol. $7, \mathrm{n}^{\circ} 4,609-622$.

PNUD (2006), Rapport mondial sur le développement humain 2006. Au-delà de la pénurie : pouvoir, pauvreté et crise mondiale de l'eau, Paris, Economica.

PRESTON S. H., VAN DE WALLE E. (1978) Urban French mortality in the nineteenth century, Population studies, vol. 32, 275-297.

ROSSIER C., SOURA A., BAYA B., COMPAORÉ G., DABIRÉ B., DOS SANTOS S. et al. (2012) The Ouagadougou Health and Demographic Surveillance System, International Journal of Epidemiology, vol. 41, $\mathrm{n}^{\circ}$ 3, 658-666

SEAGER J. (2010) Gender and water: good rhetoric, but it doesn't "count”, Geoforum, $\mathrm{n}^{\circ} 41,1-3$.

SONGSORE J., MCGRANAHAN G. (2002) Environment, wealth and health; towards an analysis of intra-urban differentials within Greater Accra Matropolitan Area, Ghana, Environment and Urbanization, vol. 5, $\mathrm{n}^{\circ} 2,10-35$.

SORENSON S. B., MORSSINK C., ABRIL CAMPOS P. (2011) Safe access to safe water in low income countries:Water fetching in current times, Social Science and Medicine, vol. 72, 1522-1526.

SUPIOT A. (2015) La gouvernance par les nombres. Cours au Collège de France 2012-2014, Paris, Fayard.

TARRASS F., BENJELLOUN M. (2012) The effects of water shortages on health and human development, Perspectives in Public Health, vol. 132, $\mathrm{n}^{\circ}$ 5, 240-244. 
TRUELOVE Y. (2011) (Re-)Conceptualizing water inequality in Delhi, India through a feminist political ecology framework, Geoforum, vol. 42, n 2, 143-152.

UNDP (2015) Human Development Report 2015. Work for Human Development, New York, United Nations Development Program.

UN-HABITAT (2005) Nairobi - Urban sector profile, Nairobi, United Nations Human Settlements Programme, 36 p.UNITED NATIONS. (2014), World Urbazination Prospects: The 2014 Revision, New York, United Nations, Population Division.

UNITED NATIONS (2005) Guide to producing statistics on time use: measuring paid and unpaid work, New York, United Nations, 400 p.

VASQUEZ W. F. (2012) Reliability perceptions and water storage expenditures: Evidence from Nicaragua, $W$ ater Resources Research, vol. 48, w10547.

WHO/UNICEF (2015) Progress on sanitation and drinking water: 2015 update and MDG assessment, Geneva, WHO/Unicef, $90 \mathrm{p}$.

WRIGHT J., GUNDRY S., CONROY R. (2004) Household drinking water in developing countries: a systematic review of microbiological contamination between source and point-of-use, Tropical Medicine and International Health, vol. 9, $\mathrm{n}^{\circ}$ 1, 106117.

$$
* * *
$$

